\title{
REVISÃO
}

\section{DISTÚRBIOS NUTRICIONAIS EM ATLETAS FEMININAS E SUAS INTER-RELAÇÕES}

\section{NUTRITION DISORDERS IN FEMALE ATHLETES AND THEIR INTERRELATIONSHIPS}

\author{
Teresa Cristina Ciavaglia VILARDI' \\ Beatriz Gonçalves RIBEIRO2 \\ Eliane de Abreu SOARES ${ }^{3}$
}

\begin{abstract}
RESUMO
Pesquisas indicam que é elevada a prevalência de distúrbios alimentares em atletas femininas jovens envolvidas em esportes que preconizam a magreza e o baixo peso corporal, tais como ginástica olímpica e corridas de longa distância. A fim de compreender a etiologia destes problemas, esta revisão apresenta os principais distúrbios nutricionais encontrados em atletas femininas, incluindo definições, critérios para diagnóstico, fatores de risco, presença de desordens inter-relacionadas - anemia, irregularidades menstruais e desmineralização óssea e suas conseqüências sobre a saúde e rendimento atlético. É fundamental ressaltar a importância da nutrição no desempenho físico. A presença de um baixo peso corporal e um consumo energético severamente restrito é o principal fator de risco para o desenvolvimento de tais distúrbios. Como as atletas e seus treinadores não apresentam adequados conhecimentos sobre nutrição, é necessária uma orientação nutricional individualizada, de forma a evitar o desenvolvimento destas alterações.
\end{abstract}

Termos de indexação: atletas, distúrbios nutricionais, anorexia, bulimia, osteoporose.

\begin{abstract}
Researches have indicated that the prevalence of nutrition disorders is high among young female athletes engaged in sports that emphasize the importance of leanness and low body weight, such as olympic gymnastics and distance running. In order to understand the etiology of these problems, this review presents the main nutrition disorders in female athletes, including definitions, diagnostic criteria, risk factors, presence of interrelated disorders (anemia, menstrual irregularities and failure of bone mineralization) and their consequences on health and athletic performance. It is fundamental to point out the importance of nutrition on physical performance. The presence of a low body weight and a very restricted energy intake is the principal risk factor for the development of nutritional disturbances. The athletes and their coaches do not have an apropriate knowledge about nutrition, so it is necessary an individualized nutritional orientation to avoid the development of these disturbances.
\end{abstract}

Index terms: athletes, nutrition disorders, anorexia, bulimia, osteoporosis.

\footnotetext{
(1) Curso de Especialização em Nutrição Clínica, Universidade Federal Fluminense.

${ }^{(2)}$ Departamento de Nutrição e Dietética, Universidade Federal do Rio de Janeiro.E-mail: beatriz@cruiser.com.br

${ }^{(3)}$ Departamento de Nutrição Básica e Experimental, Universidade do Estado do Rio de Janeiro; Departamento de Nutrição e Dietética, Centro de Ciências da Saúde Bloco J, Instituto de Nutrição, Universidade Federal do Rio de Janeiro. Cidade Universitária, Illha do Fundão, 21941-590, Rio de Janeiro, RJ, Brasil. Correspondência para/Correspondence to: E.A. SOARES.
} 


\section{INTRODUÇÃO}

Desde os primeiros Jogos Olímpicos realizados em 776 a.C. na Grécia Antiga, os quais representaram o berço da busca de relações entre nutrição e desempenho físico, atletas e treinadores buscam uma alimentação especial capaz de aumentar o rendimento físico e melhorar o desempenho (Simopoulos, 1989; Grivetti \& Applegate, 1997).

Neste final de século, apesar do crescente interesse na nutrição desportiva, ainda existe um elevado grau de desinformação, observado tanto nos atletas quanto em seus treinadores, que rotineiramente assumem a responsabilidade pelo controle dietético (Soares et al., 1994).

Devido ao desconhecimento em relação às especificidades que a prática esportiva impõe, algumas atletas comprometem a própria saúde e esforçam-se para alcançarem ou manterem uma meta inadequada de peso corpóreo, com um percentual de gordura corporal tão baixo quanto possível (Williams, 1989). Atenção especial deve ser dada às atletas adolescentes, pois durante esta fase ocorre um rápido desenvolvimento fisiológico, neurológico e psicológico (Tofler et al.,1996), estando as necessidades de nutrientes ainda mais aumentadas pelo treinamento físico intenso, estresse e ansiedade gerada pelas competições (Ribeiro, 1995).

A literatura relata que atletas jovens do sexo feminino que praticam esportes que exigem um peso corporal baixo como ginástica olímpica e corridas de longa distância possuem tipicamente uma dieta hipocalórica e um intenso gasto energético durante o treinamento físico e no próprio evento competitivo. Esta conduta pode resultar em falhas no crescimento, atraso na puberdade, esgotamento das reservas de glicogênio e fadiga (McMurray \& Anderson, 1996).

Além disso, as atletas são acometidas por uma síndrome denominada "Tríade das atletas femininas" que consiste em desordens alimentares, amenorréia e osteoporose (McArdle et al.,1999). Os componentes da tríade são inter-relacionados em etiologia, patogênese e uma série de conseqüências que incluem deficiência de ferro, anemia, irregularidades menstruais, desmineralização óssea e injúrias músculo-esqueléticas (Benson et al., 1985; Lloyd et al., 1987; Wilmore, 1991; Yeager et al.,1993; Putukian, 1994; Sundgot-Borgen, 1994a; Grooms, 1996; American College of Sports..., 1997; McArdle et al., 1999). A gênese destes distúrbios ainda é controversa, porém supõe-se que esteja relacionada ao baixo consumo energético, intenso treinamento físico, baixo percentual de gordura corpórea, alteração do perfil endócrino, ansiedade e estresse emocional (Committe on Sports Medicine, 1989).

A partir deste contexto torna-se necessária a prevenção do surgimento e desenvolvimento de complicações nutricionais e psicológicas decorrentes de todos os fatores já citados que são extremamente prejudiciais à saúde e ao desempenho das atletas podendo, muitas vezes, levá-las à morte (American College of Sports..., 1997). Portanto, o objetivo desta revisão é abordar os distúrbios nutricionais ocasionados por métodos extremos de controle de peso, freqüentemente encontrados em atletas femininas, auxiliando na maior conscientização destas atletas, seus treinadores e toda equipe de apoio.

\section{Distúrbios alimentares}

A participação de atletas femininas em esportes competitivos cresceu muito nos últimos anos. Pesquisas demonstram que o ambiente esportivo representa uma subcultura que amplia as pressões sócio-culturais pela magreza. Devido a estas pressões associadas ao esporte, as adolescentes se tornam mais susceptíveis aos distúrbios alimentares, principalmente em esportes que preconizam o baixo peso corporal como ginástica olímpica e corridas de longa distância (Petrie \& Stoever, 1993; Sundgot-Borgen, 1994a; Marshall \& Harber, 1996).

A ginástica olímpica é um esporte caracterizado pela leveza dos movimentos, pela arte do equilíbrio, flexibilidade e pelo domínio do corpo. Devido a isso preconiza-se que ginastas sejam mais leves e mais magras do que outras meninas da mesma idade. Em se tratando de corridas de longa distância, atletas que apresentam um menor peso corporal e diminuída percentagem de gordura corpórea estariam em vantagem em relação às outras, pois neste esporte a atleta tem que conduzir seu corpo a uma longa distância em velocidade máxima (Bale, 1994).

De acordo com Diagnostic and Statistical Manual of Mental Disorders (American Psychiatric..., 1987; American Psychiatric..., 1994), os distúrbios alimentares são caracterizados por alterações no comportamento alimentar incluindo anorexia e bulimia nervosa, sendo esses mais freqüentemente encontrados em atletas femininas.

A anorexia nervosa é caracterizada por uma extrema restrição energética auto-imposta tendo como objetivo a perda excessiva de peso (Ruud \& Grandjean, 1996). Os sintomas mais comuns deste distúrbio incluem: manutenção do peso corporal inferior a $85 \%$ do que é considerado adequado para estatura e idade, intenso medo de engordar, percepção alterada da imagem corporal, distúrbios menstruais, desmineralização óssea, perda de massa muscular e gordura corporal, irregularidades digestivas, arritmias cardíacas, desidratação, intolerância ao frio (mãos e pés), cabelos finos e fracos, entre outras (Sundgot-Borgen \& Corbin, 1987; Wichmann \& Martin, 1993; Katch \& McArdle, 1996). Deve-se ressaltar, que uma significante restrição energética acarreta uma diminuição na taxa metabólica basal e prejuízos nas funções músculo-esquelética, cardiovascular, endócrina, termoregulatória e outras (American College of Sports..., 1997). 
A bulimia nervosa está relacionada a uma ingestão descontrolada e compulsiva geralmente seguida por uma purgação. Os métodos de purgação freqüentemente encontrados, os quais estão relacionados a um comportamento patológico de controle de peso incluem: indução a vômitos, abuso no uso de laxantes, diuréticos e moderadores de apetite e prática de exercícios físicos intensos (Ruud \& Grandjean, 1996). As principais conseqüências da bulimia nervosa são: perda de fluidos e eletrólitos durante a purgação podendo levar à desidratação, desequilíbrio ácido-básico e eletrolítico e arritmias cardíacas (American College of Sports..., 1997). Algumas atletas que induzem o vômito após episódios de compulsão alimentar podem apresentar uma diminuição na concentração do potássio sérico, que é o principal cátion responsável pela contração muscular. A fraqueza muscular característica de tais atletas pode estar relacionada à hipocalemia (Zucker et al., 1985). A indução a vômitos também pode resultar em problemas físicos crônicos incluindo, distúrbios gastrintestinais; aumento da glândula parótida; erosão e perda do esmalte dentário; desidratação entre outros (Yurth, 1995; Katch \& McArdle, 1996; American College of Sports, 1997).

Segundo o Diagnostic and Statistical Manual of Mental Disorders (Americam Psychitric..., 1987; Americam Psychiatric..., 1994)) existe uma distinção entre distúrbios alimentares clinicamente diagnosticados (anorexia e bulimia nervosa) e comportamento patológico de controle de peso. Muitas atletas apresentam sinais de comportamento alimentar alterado, porém não estão enquadradas no diagnóstico de distúrbios alimentares. Observa-se que estas atletas também estão em risco de desenvolver sérios problemas de saúde incluindo depleção do glicogênio muscular, desidratação, perda de massa muscular, hipoglicemia, anormalidades eletrolíticas, anemia, amenorréia e osteoporose, necessitando de avaliação e tratamento (Wichmann \& Martin, 1993; Yurth, 1995; Marshall \& Harber, 1996; American College of Sports..., 1997).

Além destes clássicos distúrbios, uma condição prevalente entre atletas é a "Anorexia Atlética". Os critérios para seu diagnóstico incluem: perda de peso, atraso na puberdade, disfunção menstrual, queixas gastrintestinais, ausência de doença ou desordem afetiva que pudesse explicar a redução de peso, falsa imagem corporal, excessivo medo de ganhar peso, restrição alimentar, vômitos auto-induzidos, uso de laxantes, diuréticos e exercícios físicos compulsivos (Sundgot-Borgen, 1994b).

A maioria das atletas que querem perder peso não apresentam conhecimentos suficientes sobre nutrição e métodos apropriados de controle ponderal e não seguem uma orientação ou supervisão, adquirindo informações de outras atletas, treinadores ou revistas não especializadas.

Em uma pesquisa realizada por Rosen \& Hough (1988), foi verificado que a maioria dos treinadores estabeleciam medidas de controle de peso para suas atletas, mesmo sabendo muito pouco sobre nutrição e sua relação com o rendimento. Observou-se ainda, que 75\% das ginastas que foram advertidas por seus treinadores para perderem peso, apresentaram comportamento patológico de controle de peso.

Da mesma forma, Loosli et al. (1986) realizaram um estudo com 97 ginastas adolescentes competitivas, tendo como objetivo investigar os conhecimentos das atletas sobre nutrição. As informações foram obtidas através de um questionário que envolveu questões sobre composição dos alimentos e como tais alimentos se relacionavam com o desempenho atlético. Os pesquisadores classificaram como inadequados os conhecimentos nutricionais das ginastas, observando a importância de programas de educação nutricional para o grupo estudado.

O risco para o desenvolvimento de distúrbios alimentares é maior se a restrição alimentar não for supervisionada por um profissional experiente. Como já ressaltado anteriormente, o perigo destas dietas é que normalmente as necessidades energéticas não são alcançadas, estando aumentadas pelo treinamento físico e, em se tratando de atletas adolescentes, as necessidades de nutrientes são ainda maiores (Kopp-Woodroffe et al., 1999).

O consumo inadequado de atletas vem sendo confirmado a partir dos estudos dietéticos realizados por diferentes pesquisadores. Chen et al. (1989) realizaram uma pesquisa com atletas, tanto de elite quanto amadores. Dentro desse grupo encontrava-se 5 ginastas femininas de elite. O objetivo foi detectar problemas nutricionais possivelmente existentes e fornecer informações científicas para prevenir seu surgimento. As informações dietéticas foram obtidas através do método de pesagem de alimentos por um período de 3 a 5 dias. A ingestão energética média foi de $2298 \mathrm{kcal} / \mathrm{dia}$. As percentagens de glicidios, proteínas e lipídios foram respectivamente $42 \%$, $16 \%$ e $42 \%$, evidenciando-se um consumo aumentado de proteínas e lipídios em detrimento de uma baixa ingestão de glicídios na dieta. Os autores destacaram a importância da educação nutricional para atletas e treinadores.

Ratificando esta pesquisa, Ribeiro (1995) realizou um estudo com 54 ginastas adolescentes, pertencentes a clubes e academias da cidade do Rio de Janeiro e São Paulo com o objetivo de avaliar o consumo alimentar de atletas de ginástica olímpica. Os método de avaliação dietética utilizados foram: recordatório de 24 horas e o registro alimentar de 3 dias. Os resultados demonstraram que o consumo energético das atletas cariocas e paulistas, de 11 a 14 anos, estava abaixo das recomendações americanas (National Council..., 1989) para adolescentes. A ingestão glicídica contribuiu com $49 \%$ a $59 \%$ do valor energético total, menores do que o recomendado para atletas competitivas (60-65\%). Além disso, os hábitos dietéticos das atletas estudadas mostraram-se similares aos de adolescentes não atletas caracterizados por um 
elevado consumo de alimentos com baixa densidade nutricional, como balas, chicletes e refrigerantes.

Observou-se com estes estudos que as atletas têm um baixo consumo energético frente as demandas necessárias à prática desportiva. Além disso, suas dietas normalmente encontram-se em desequilíbrio com relação aos macronutrientes, o que implicaria ingestão precária de vitaminas e minerais.

Diante destas evidências, torna-se clara a necessidade de orientação individualizada para adequar os hábitos alimentares das atletas, bem como uma educação nutricional para melhorar o nível de conhecimento das próprias atletas e seus treinadores. Estudos sobre conhecimentos em nutrição e consumo dietético proporcionam um ponto de partida para a educação nutricional destes indivíduos (Short, 1996).

\section{Deficiência de ferro e anemia esportiva}

As reservas corporais de ferro são essenciais para as vias metabólicas e para a produção de energia pelos músculos. A depleção das reservas corporais, que reduz as concentrações de mioglobina e dos citocromos, pode deteriorar o metabolismo aeróbio e limitar a capacidade de executar exercícios. Existe uma ampla evidência de que a anemia ferropriva pode causar uma diminuição na capacidade aeróbia ( $\mathrm{VO}_{2}$ máx.), redução do trabalho físico, diminuição da resistência e aumento da fadiga (Risser et al., 1988; Rowland \& Kelleher, 1989; Haymes, 1996).

O método mais preciso para avaliação da quantidade de ferro orgânico é através da biopsia da medula óssea, porém, trata-se de um procedimento invasivo e caro. As concentrações de ferritina sérica são diretamente proporcionais às reservas orgânicas de ferro (Nuviala \& Lapieza, 1997). Entretanto, torna-se necessário uma avaliação completa do estado de ferro para o diagnóstico de deficiência deste mineral. Vários parâmetros sangüíneos, além das concentrações de ferritina sérica, são utilizados para medir o estado de ferro como: a saturação de transferrina $(\mathrm{ST})$, a hemoglobina $(\mathrm{Hb})$, o hematócrito $(\mathrm{Ht})$, a capacidade de conjugação de ferro total (CCFT) e o ferro sérico (Fe) (Ruud \& Grandjean, 1996). É importante observar que uma deficiência de ferro se desenvolve de maneira gradual antes da anemia se tornar evidente.

Estudos mostram que atletas, especialmente as de resistência, tendem a desenvolver uma condição conhecida como "anemia esportiva" ou "pseudoanemia", que pode ocorrer no início de um programa de treinamento ou durante um treinamento prolongado. Reduções transitórias na concentração de hemoglobina, na contagem de hemácias e hematócrito, que caracterizam esta condição, podem ser devidas à expansão do volume plasmático como uma adaptação fisiológica aos exercícios extenuantes (Williams, 1989; Weight et al., 1992; Nachtigall et al., 1996). Tal aumento no volume plasmático pode ser benéfico devido ao decréscimo na resistência ao fluxo sangüíneo, melhora na sudorese e um maior volume de ejeção (Haymes, 1996). Entretanto, pesquisas com atletas de resistência, do sexo feminino, revelam que estas não apresentam apenas uma redução na concentração de hemoglobina relacionada a uma hemodiluição, mas também um estado de deficiência de ferro com ou sem anemia.

As causas mais prováveis de depleção deste mineral são: ingestão quantitativa insuficiente de ferro; redução na absorção deste nutriente devido a dietas com baixa biodisponibilidade; perda através da transpiração e menstruação; perda gastrintestinal de sangue levando à excreção através das fezes, perda pela urina e hemólise intravascular (Williams, 1989; McArdle et al., 1992; Telford et al., 1993; Moore et al., 1995; Haymes, 1996; Nachtigall et al., 1996).

Rowland \& Kelleher (1989) ressaltaram que o consumo inadequado de ferro parece ser o fator mais importante para o desenvolvimento de uma deficiência deste mineral. Além disso, as atletas adolescentes encontram-se em risco freqüente de desenvolver esta condição devido ao aumento de suas necessidades fisiológicas (Ruud \& Grandjean, 1996).

Loosli et al. (1986) realizaram um estudo com 97 ginastas adolescentes competitivas, tendo como objetivo avaliar a qualidade da dieta consumida pelas atletas. As informações dietéticas foram obtidas através do registro alimentar de 3 dias. Com relação à ingestão energética, as ginastas consumiam em média 1838 kcal diárias e 40\% ou mais consumiam dietas que contribuíram com menos de dois terços das recomendações para cálcio, ácido fólico, piridoxina, ferro e zinco.

Atletas femininas que consomem dietas com baixa biodisponibilidade de ferro, como por exemplo, dietas vegetarianas, podem se encontrar em risco de uma deficiência devido à baixa ingestão de ferro heme (Nuviala \& Lapieza, 1997). Snyder et al. citados por Nuviala et al. (1996) realizaram um estudo com corredoras que consumiam dieta vegetariana modificada (menos de 100 g de carne por semana) comparando-as com as que consumiam carne vermelha. Não foi observada diferença no consumo energético total e ambos os grupos de corredoras consumiram aproximadamente $14 \mathrm{mg}$ de ferro dietético. No entanto, os autores relataram que a biodisponibilidade deste mineral encontrou-se significativamente $(p<0,05)$ mais baixa nas dietas de corredoras vegetarianas, sendo que estas apresentaram concentrações séricas de ferritina mais baixas do que o outro grupo de corredoras. Os pesquisadores concluíram que hábitos alimentares vegetarianos poderiam agravar a deficiência de ferro.

A perda de ferro através da transpiração excessiva também pode estar relacionada ao balanço negativo deste mineral. Atletas que treinam exaustivamente podem 
eliminar 1 a 2 litros de suor por hora, perdendo também uma quantidade substancial de ferro (Williams, 1989; Telford et al.,1993). Contudo, parte do ferro perdido no suor pode ser proveniente da descamação cutânea (Haymes, 1996).

Observa-se que corredoras de longa distância podem apresentar uma perda de ferro através de sangramento gastrintestinal. A presença de sangue nas fezes pode ser devido a vasoconstrição dos vasos esplâncnicos durante o exercício intenso. Após corridas de longa distância, estas atletas também podem apresentar aumento na contagem de hemácias na urina. Uma possível causa desta hematúria é o trauma na parede vesical durante a corrida. Como a quantidade de ferro encontrada na urina é relativamente pequena, é pouco provável que esta seja a principal fonte de perda deste mineral (Haymes, 1996).

Uma concentração reduzida de hemoglobina em atletas também pode ocorrer devido à hemólise intravascular, devido ao trauma mecânico imposto aos capilares dos pés, principalmente de corredoras, provocado pelos impactos repetitivos (Williams, 1989; Moore et al., 1995; Telford et al.,1993).

Diante dessas evidências, fica claro que o consumo alimentar inadequado associado a uma prática esportiva intensa contribuem para a deficiência orgânica de ferro em atletas.

\section{Irregularidades menstruais}

Atletas femininas que praticam exercícios físicos intensos e apresentam alterações no comportamento alimentar, além de correrem o risco de desenvolverem deficiência de ferro, com ou sem anemia, comumente apresentam irregularidades menstruais caracterizadas por oligomenorréia e amenorréia. O ciclo menstrual regular ou eumenorréico varia de 23 a 35 dias, com 10 a 13 ciclos por ano. Oligomenorréia está relacionada à presença de 3 a 6 ciclos por ano com intervalos maiores do que 36 dias. Amenorréia é definida como a falta ou interrupção do fluxo menstrual, com menos de 2 ciclos menstruais por ano. Amenorréia primária corresponde à falta de períodos menstruais em meninas de até 16 anos de idade. Amenorréia secundária está relacionada à interrupção de 3 a 12 períodos menstruais consecutivos em jovens que apresentaram uma menarca normal (Putukian, 1994).

O equilíbrio endócrino que regula as funções reprodutoras femininas pode ser alterado por fatores genéticos, psicológicos e físicos. O treinamento físico intenso pode estar associado à presença de irregularidades menstruais. Atletas femininas que desempenham exercícios físicos intensos são as que mais apresentam tais irregularidades (Arena et al., 1995).

Muitos fatores encontram-se associados à amenorréia atlética. Os freqüentemente citados são: distúrbios no comportamento alimentar, restrição dietética, intensidade dos treinamentos, estresse psicológico e fisiológico, treinamento físico antes da menarca, retardo do início da função menstrual, baixo peso corporal e baixo percentual de gordura corpórea (Warren, 1992; Bale, 1994; O' Connor et al. 1996b; Ruud \& Grandjean, 1996).

Com relação ao percentual de gordura corporal, muitos autores questionam a teoria da "gordura crítica", a qual preconiza que o "nível crítico" de gordura corporal para iniciar e manter os ciclos menstruais regulares seja em torno de 17\% (Katch \& McArdle, 1996). Entretanto, algumas atletas com baixa percentagem de gordura corporal (menor de $17 \%$ ) apresentam ciclos menstruais regulares (Ruud \& Grandjean, 1996). Dados mais recentes sugerem que a composição corporal e a distribuição regional de gordura estão mais relacionadas ao início dos distúrbios menstruais do que à gordura corporal total (Arena et al.,1995; Brownel et al. citados por Ruud \& Grandjean, 1996). Acredita-se ainda que o tecido adiposo é um sítio de conversão de andrógenos em estrógenos (Bale et al., 1996; Katch \& McArdle, 1996).

O estresse psicológico e fisiológico induzido pela competição e pelo treinamento físico intenso pode aumentar a produção de andrógenos adrenais e modificar o clearance metabólico de estrógenos, exercendo um papel importante na etiologia da amenorréia (Warren, 1992; Bale, 1994). Os principais hormônios envolvidos são: hormônio luteinizante, estradiol, prolactina, endorfina, prostaglandinas, dopamina, epinefrina, norepinefrina e cortisol (Putukian, 1994).

Arena et al. (1995) observaram um aumento significativo nas concentrações plasmáticas de hormônios anti-reprodutivos (melatonina, prolactina, ß-endorfina, ßlipotrofina e cortisol), epinefrina e norepinefrina, em atletas jovens que realizavam programas de treinamento físico moderado. Concentrações aumentadas de cortisol e norepinefrina podem modificar o turnover dos neurotransmissores cerebrais alterando seu transporte através da barreira hematoencefálica e inibindo a liberação do hormônio luteinizante (Laughlin \& Yen, 1996).

Além disso, o aumento na secreção de cortisol induzido pelo estresse físico pode ser potencializado por uma severa restrição energética (Arena et al.,1995). As atletas que treinam em tempo integral e sofrem inúmeras pressões no que diz respeito ao controle de peso corporal são mais propensas a desenvolverem irregularidades menstruais (Bale et al., 1996). Baer \& Taper (1992) realizaram uma pesquisa com 6 corredoras adolescentes amenorréicas e 6 eumenorréicas com o objetivo de avaliar a relação entre o consumo dietético e o treinamento físico. As atletas preencheram registros alimentares de 7 dias e um questionário pessoal. Foi observado que as corredoras amenorréicas corriam em média 64 quilômetros por semana, consumiam em média 1912 kcal/dia e ingeriam uma quantidade de ferro menor que $70 \%$ da recomendação (National Council..., 1989). As corredoras eumenorréicas corriam 32 quilômetros por semana, ingeriam 1644 kcal/dia 
e consumiam menos de $70 \%$ da recomendação para cálcio, ferro, magnésio e zinco. As corredoras amenorréicas necessitavam de um aporte energético maior por dia para manterem o rendimento atlético. Os autores observaram que corredoras adolescentes que treinavam intensamente e consumiam dieta hipocalórica apresentavam maior incidência de amenorréia secundária e concentrações reduzidas de estradiol.

Atletas de resistência tendem a desenvolver déficit energético devido a ingestão abaixo do que é necessário para sustentar o elevado gasto de energia relacionado ao exercício físico intenso. A incidência de amenorréia entre estas atletas está mais associada ao balanço energético negativo do que ao exercício por si só (Laughlin \& Yen, 1996; American College Sports..., 1997).

Wilmore et al. (1992) avaliaram 13 corredoras (8 amenorréicas e 5 eumenorréicas) com o objetivo de investigar uma possível conservação de energia através de uma redução na taxa metabólica de repouso em corredoras amenorréicas comparadas às eumenorréicas. As informações dietéticas foram obtidas através do registro alimentar de 3 dias. Foi observado que o grupo das corredoras amenorréicas consumia em média 1781 kcal e o grupo das eumenorréicas consumia 1690 kcal diárias. Os pesquisadores concluíram que ambos os grupos apresentavam uma ingestão energética total significativamente menor do que era esperado para idade, altura e nível de atividade física. Nenhuma evidência de conservação de energia foi observada no repouso após as refeições ou durante o exercício que pudesse explicar o déficit energético nas corredoras.

Apesar de muitos autores considerarem a presença de amenorréia como uma adaptação normal do organismo a períodos de restrição alimentar e intenso treinamento físico, vários estudos mostram que se trata de um sintoma indicativo de um sério problema clínico (Skolnick, 1993). A conseqüência clínica mais prejudicial da amenorréia atlética é seu impacto sobre o esqueleto (Voss et al., 1998).

O perfil endócrino das atletas amenorréicas é caracterizado por decréscimo na produção de estrogênio. Acredita-se que o estrogênio promova a absorção de cálcio pelos ossos (Yurth, 1995) e diminua a sensibilidade óssea ao paratormônio. Quando ocorre deficiência de estrogênio, os ossos se tornam mais sensíveis à ação do paratormônio (Frederick \& Hawkins, 1992).

Foi descoberto que os osteoblastos possuem receptores para o estrogênio e o mecanismo de remodelação óssea envolve tal hormônio. O estrogênio pode agir diretamente sobre seu receptor para estimular a remodelação óssea influenciando as secreções dos osteoblastos que por sua vez, agem sobre a matriz extracelular promovendo a mineralização (Warren, 1992).

As conseqüências a longo prazo de um estado hipoestrogênico crônico em atletas jovens incluem a perda de massa óssea, muitas vezes irreversível (Yeager et al.,
1993; Constantini, 1994; Putukian, 1994; Yurth, 1995; O'Connor et al., 1996b). Atletas jovens amenorréicas podem apresentar uma massa óssea menor do que as que sempre mantiveram ciclos menstruais normais, menor até mesmo do que muitas mulheres na pós-menopausa e consequentemente um risco aumentado de osteoporose.

Outras conseqüências clínicas observadas em atletas amenorréicas estão relacionadas à saúde reprodutiva e ao risco aumentado de doenças cardiovasculares. A redução na fase lútea é um achado comum entre as atletas e a incidência de infertilidade é elevada. A prática de atividade física regular e a concentração plasmática de estradiol estão envolvidos na prevenção de coronariopatias, principalmente através do efeito benéfico sobre as lipoproteínas plasmáticas (HDL colesterol). Um estado hipoestrogênico crônico pode afetar negativamente os lipídios plasmáticos anulando os efeitos benéficos do exercício físico sobre os mesmos (Constantini, 1994).

Sendo assim, a amenorrréia em atletas deve ser prevenida e quando presente ser criteriosamente investigada e revertida, através de uma redução do treinamento e do aumento da ingestão energética, frente as inúmeras consequências negativas à saúde associadas a esta disfunção.

\section{Osteoporose}

Osteoporose é uma condição clínica definida como um decréscimo na massa óssea (matriz osteóide e componente macrocristalino inorgânico) (Putukian, 1994). Associa-se a redução na densidade minerálica óssea principalmente da coluna vertebral e do fêmur proximal e a maior incidência de injúrias músculo-esqueléticas (Putukian, 1994; Ruud \& Grandjean, 1996).

Acredita-se que a amenorréia é o principal fator de risco para a redução da massa óssea (Wilmore, 1991). São achados freqüentes em atletas amenorréicas: incapacidade de alcançar o pico de massa óssea, perda prematura de massa óssea e osteoporose (Constantini, 1994).

O pico de massa óssea é o principal determinante da quantidade óssea total (Düppe et al.,1997), sendo medido e expresso como densidade minerálica óssea (DMO) $(\mathrm{g} / \mathrm{cm} 2)$, devido à elevada correlação existente entre DMO e força muscular (Vuori, 1996).

Vários fatores encontram-se relacionados ao pico de massa óssea incluindo: fatores hormonais (concentrações reduzidas de estrógenos; estradiol e estrona; níveis reduzidos de andrógenos; níveis aumentados de cortisol; fatores nutricionais (estado nutricional, composição corporal, distúrbios nutricionais, baixa ingestão energética e de cálcio, elevada ingestão de proteínas e fibras); fatores genéticos e outros (exercícios físico, fumo e álcool) (Snead et al., 1992; Constantini, 1994; Lichtenbelt et al., 1995; Heaney, 1996; Vuori, 1996; Dook, 1997; Düppe et al., 1997). 
A prevenção primária da osteoporose está relacionada à obtenção de densidade minerálica óssea máxima através da ingestão adequada de cálcio, principalmente durante a infância e adolescência (Putukian, 1994). A idade precisa em que se alcança a maximização do pico de massa óssea varia, porém acredita-se que para o fêmur proximal e corpos vertebrais seja em torno dos 18 anos de idade (Vuori, 1996).

De acordo com Grooms (1996), atletas femininas que não atingiram seu pico máximo de massa óssea na adolescência poderão apresentar perda óssea prematura e risco de osteoporose precoce. Esta condição não apenas prejudica o rápido crescimento ósseo característico da adolescência como também acelera o processo de perda óssea numa idade mais avançada (Mehler, 1996; Voss et al., 1998).

Estudos mostram que os efeitos da atividade física sobre a densidade minerálica óssea podem ser tanto maléficos quanto benéficos (Düppe et al., 1997). Muitas atletas jovens apresentam densidade minerálica equivalente a idosas de 70 ou 80 anos, sendo comum a expressão "ossos velhos em atletas jovens" (Skolnick, 1993). Alguns autores ratificam que atletas que praticam exercícios físicos intensos e desenvolvem amenorréia hipoestrogênica tendem a apresentar densidade minerálica óssea menor do que o esperado, aumentando o risco de uma osteoporose precoce (Warren, 1992; Robinson et al., 1995).

Existe um consenso na literatura de que a prática regular de exercícios de carga (musculação) exerce efeitos benéficos sobre a massa óssea (Warren, 1992; Lee et al., 1995; Robinson et al., 1995; Dook, 1997).

O exercício promove o aumento da massa óssea através do estresse físico sobre os ossos corticais favorecendo a remodelação óssea (Putukian, 1994). Carter citado por Lee et al. (1995) demonstrou que a resposta óssea ao estresse é sítio específica, ou seja, cada parte do esqueleto apresenta seu próprio limite para adaptação.

Vários pesquisadores também sugerem que o exercício físico esteja associado à hipertrofia do tecido muscular, demonstrando existir relação significativa entre a força muscular, percentagem de massa magra e densidade minerálica óssea (Dook,1995; Lee et al., 1995).

As atletas que estão envolvidas em atividades de alto impacto e exercícios de carga podem apresentar densidade minerálica óssea maior do que outras que não estão envolvidas nestes tipos de atividades (Dook et al., 1997; Lee et al., 1995). Além disso, é provável que a prática destes exercícios possa proteger atletas amenorréicas contra uma maior perda óssea $\left(\mathrm{O}^{\prime}\right.$ Connor et al., 1996a.).

Robinson et al. (1995) realizaram um estudo com 20 corredoras e 21 ginastas de 17 a 27 anos de idade. Os pesquisadores observaram que as ginastas apresentavam densidade minerálica óssea maior do que as corredoras, apesar da prevalência similar de amenorréia e oligomenorréia (47\% para ginastas e $30 \%$ para corredoras).
Os resultados observados neste estudo sugeriram que a atividade física de alto impacto realizada pelas ginastas estimularia mais o crescimento da massa óssea do que a atividade de baixo impacto desempenhada pelas corredoras, ou seja, as forças mecânicas extremas geradas pelo treinamento das ginastas competitivas seriam elevadas o suficiente para compensar o estado hipoestrogênico encontrado nas atletas amenorréicas. Além disso, os autores também concluíram que, como a percentagem de gordura corporal não apresentou diferença entre os dois grupos de atletas, a presença de uma elevada percentagem de massa magra e força muscular observada nas ginastas poderia ter contribuído para o aumento da densidade minerálica óssea.

\section{CONSIDERAÇÕES FINAIS}

De acordo com o que foi descrito, jovens do sexo feminino que praticam esportes que exigem baixa massa corporal como ginástica olímpica e corridas de longa distância, normalmente consomem dietas com reduzido valor energético. A presença de um consumo severamente restrito é um dos principais fatores de risco relacionado ao desenvolvimento de distúrbios alimentares, deficiência de ferro, irregularidades menstruais e desmineralização óssea que pode levar até mesmo à osteoporose.

Como as atletas e seus treinadores não apresentam conhecimentos adequados sobre nutrição, geralmente as atletas comprometem a própria saúde esforçando-se para alcançarem ou manterem uma meta inadequada de peso corpóreo, ou seja, um percentual de gordura corporal tão baixo quanto possível.

Devido a isso, torna-se necessário uma educação nutricional direcionada às atletas, seus treinadores e à equipe de apoio para que os mesmos se conscientizem dos efeitos adversos dos métodos extremos de controle de peso sobre a saúde destas próprias atletas.

\section{REFERÊNCIAS BIBLIOGRÁFICAS}

AMERICAN COLLEGE OF SPORTS MEDICINE. The female athlete triad. Medicine and Science in Sports and Exercice, Baltimore, v.29, n.5, p.1-9, 1997.

AMERICAN PSYCHIATRIC ASSOCIATION. Diagnostic and statistical manual of mental disorders. 3.ed. Rev. Washington DC, 1987. p.65-69.

AMERICAN PSYCHIATRIC ASSOCIATION. Diagnostic and statistical manual of mental disorders. 4.ed. Washington DC, 1994. p.1-2.

ARENA, B., MAFFUlLi, N., MAFFUlLI, F., MORLEO, M.A. Reproductive hormones and menstrual changes with exercise in female athletes. Sports Medicine, Auckland, v.19, n.4, p.278-287, 1995. 
BAER, J.T., TAPER, J. Amenorrheic and eumenorrheic adolescent runners: dietary intake and exercise training status. Journal of the American Dietetic Association, Chicago, v.92, n.1, p.8991, 1992.

BALE, P. Body composition and menstrual irregularities of female athletes. Are they precursors of anorexia? Sports Medicine, Auckland, v.17, n.6, p.347-352, 1994.

BALE, P., DOUST, J., DAWSON, D. Gymnasts, distance runners, anorexics body composition and menstrual status. The Journal of Sports Medicine and Physical Fitness, Torino, v.36, n.1, p.49-53, 1996.

BENSON, J., GILLIEN, D.M., BOURDET, K., LOOSLI, A.R. Inadequate nutrition and chronic calorie restriction in adolescent ballerinas. The Physician and Sportsmedicine, v.13, n.10, p.79-90, 1985.

CHEN, J.D., WANG, J.F., LI, K.J., ZHAO, Y.W., WANG, S.W., JIAO, Y., HOU, X.Y. Nutritional problems and measures in elite and amateur athletes. American Journal of Clinical Nutrition, Bethesda, v.49, n.5, p.1084-1089, 1989.

COMMITTE ON SPORTS MEDICINE. Amenorrhea in adolescent athletes. Pediatrics, Evanston, v.84, n.2, p.394-395, 1989.

CONSTANTINI, N.W. Clinical consequences of athletic amenorrhea. Sports Medicine, Auckland, v.17, n.4, p. 213-223, 1994.

DOOK, J.E. Exercise and bone mineral density in mature female athletes. Medicine and Science in Sports and Exercise, Baltimore, v.29, n.3, p.291-296, 1997.

DÜPPE, H., GARDSELL, P., JOHNELL, O., NILSSON, B.E., RINGSBERG, K. Bone mineral density, muscle strength and physical activity. a population based study of 332 subjects aged 15-42 years. Acta Orthopaedica Scandinavica, Copenhagen, v.68, n.2, p.97-103, 1997.

FREDERICK, L., HAWKINS, S.T. Comparison of nutrition knowledge and attitudes, dietary practices and bone densities of postmenopausal women, female college athletes and nonathletic college women. Journal of the American Dietetic Association, Chicago, v.92, n.3, p.299-305, 1992.

GRIVETTI, L.E., APPLEGATE, E.A. From Olympia to Atlanta: a cultural-historical perspective on diet and athletic training. Journal of Nutrition, Bethesda, v.127, p.860S-868S, 1997. Supplement 5.

GROOMS, A.M. The female athlete triad. Journal of the Florida Medical Association, Jacksonville, v.83, n.7, p.479-481, 1996.

HAYMES, E.M. Minerais de traço e exercícios. In: WOLINSKY, I., HICKSON JR, J.F. Nutrição no exercício e no esporte. 2.ed. São Paulo : Roca, 1996. p.242-257.

HEANEY, R.P. Bone mass, nutrition and other lifestyle factors. Nutrition Reviews, New York, v.54, n.4, p.3-10, 1996.

KATCH, F.I., McARDLE, W.D. Nutrição, exercício e saúde. 4.ed. Rio de Janeiro : MEDSI, 1996. 657p.

KOPP-WOODROFFE, S.A., MANORE, M.M., DUESK, C.A., SKINNER, J.S., MATT, K.S. Energy and nutrient status of amenorrheic athletes participating in a diet and exercise training intervention program. International Journal of Sport Nutrition, Champaign, v.9, n.1, p.70-88, 1999.

LAUGHLIN, G.A., YEN, S.S.C. Nutritional and endocrinemetabolic aberrations in amenorrheic athletes. Journal of
Clinical Endocrinology and Metabolism, Baltimore, v.81, n.12, p.4301-4309, 1996.

LEE, E.J., LONG, K.A., RISSER, W.L., POINDEXTER, H.B.W., GIBBONS, W.E. \& GOLDZIEHER, J. Variations in bone status of contralateral and regional sites in young athletic women. Medicine and Science in Sports and Exercise, Baltimore, v.27, n.10, p.1354-1361, 1995.

LICHTENBELT, W.D.V.M., FOGELHOLM, M., OTTENHEIJM, R., WESTERTERP, K.R. Physical activity, body composition and bone density in ballet dancers. British Journal of Nutrition, London, v.74, n.4, p.439-451, 1995.

LLOYD, T., BUCHANAN, J.R., BITZER, S., WALDMAN, C., MYERS, C. FORD, B.G. Interrelationships of diet, athletic activity, menstrual status and bone density in collegiate women. American Journal of Clinical Nutrition, Bethesda, v.46, n.4, p.681-684, 1987.

LOOSLI, A.R., BENSON, J., GILLIEN, D.M. \& BOURDET, K. Nutrition habits and knowledge in competitive adolescent female gymnasts. The Physician and Sportsmedicine, v.14, n.8, p.118-130, 1986.

MARSHALL, J.D., HARBER, V.J. Body dissatisfaction and drive for thinness in high performance field hockey athletes. International Journal of Sports Medicine, Stuttgart, v.17, n.7, p.541-544, 1996.

McARDLE, W.D., KATCH, F.I., KATCH, V.L. Fisiologia do exercício: energia, nutrição e desempenho humano. 3.ed. Rio de Janeiro: Guanabara Koogan, 1992. 510p.

McARDLE, W.D., KATCH, F.I., KATCH, V.L. Sports and exercise nutrition. Maryland : Lippincott Willians \& Wilkins, 1999. 750p.

MCMURRAY, R.G., ANDERSON, J.J.B. Introdução à nutrição no exercício e no esporte. In: WOLINSKY, I., HICKSON JR., J.F. Nutrição no exercício e no esporte. 2.ed. São Paulo : Roca, 1996. p.2-14.

MEHLER, R.S. Bone density in amenorrheic athletes and in anorexia nervosa (letter). Jama, Chicago, v.276, n.17, p.1384-1385, 1996.

MOORE, R.J. FRIEDL, K.E., TULLEY, R.T., ASKEW, E.A. Maintenance of iron status in healthy men during an extended period of stresse and physical activity. American Journal of Clinical Nutrition, Bethesda, v.58, n.6, p.923-927, 1995.

NACHTIGALLL, P., NIELSEN, P., FISHER, R., ENGELHARDT, R., GABBE, E.E. Iron deficiency in distance runners : a reinvestigation using Fe-labelling and non-invasive liver iron quantification. International Journal of Sports Medicine, Stuttgart, v.17, n.7, p.473-479, 1996.

NATIONAL RESEARCH COUNCIL (USA). Food and nutrition board: recommend dietary allowances. 10.ed. Washington DC : National Academy Sciences, 1989. 284p.

NUVIALA, R.J., CASTILLO, M.C., LAPIEZA, M.G., ESCANERO, J.F. Iron nutritional status in female karatekas, handball and basketball players and runners. Physiology and Behavior, Elmsford, v.59, n.3, p.449-453, 1996.

NUVIALA, R.J., LAPIEZA, M.G. Disparity between diet and serum ferritin in elite sportswomen. Nutrition Research, v.17, n.3, p.451-461, 1997.

O'CONNOR, P.J., LEWIS, R.D., BOYD, A. Health concerns of artistic women gymnasts. Sports Medicine, Auckland, v.21, n.5, p.321-325, 1996a. 
O'CONNOR, P.J., LEWIS, R.D., KIRCHNER, E.M., COOK, D.B. Eating disorder symptoms in former female college gymnasts: relation with body composition. American Journal of Clinical Nutrition, Bethesda, v.64, n.6, p.840-843, 1996b.

PETRIE, T.A., STOEVER, S. The incidence of bulimia nervosa and pathogenic weight control behaviors in female collegiate gymnasts. Research Quarterly for Exercise and Sport, Washington DC, v.64, n.2, p.238-241, 1993.

PUTUKIAN, M. The female triad: eating disorders, amenorrhea and osteoporosis. Medical Clinics of North America, Philadelphia, v.78, n.2, p.345-356, 1994.

RIBEIRO, B.G. Avaliação nutricional de ginastas competitivas de ginástica olímpica. Rio de Janeiro, 1995. 110p. Dissertação (Mestrado em Nutrição Humana) - Instituto de Nutrição, Universidade Federal do Rio de Janeiro, 1995.

RISSER, W.L., LEE, E.J., POINDEXTER, H.B.W., WEST, M.S., PIVARNIK, J.M., RISSER, J.M.H., HICKSON, J.F. Iron deficiency in female athletes: its prevalence and impact on performance. Medicine and Science in Sports and Exercise, Baltimore, v.20, n.2, p.116-121, 1988.

ROBINSON, T.L., SNOW-HARTNER, C., TAAFFE, D.R., GILLIS, D., SHAW, J., MARCUS, R. Gymnasts exhibit higher bone mass than runners despite similar prevalence of amenorrhea and oligomenorrhea. Journal of Bone and Mineral Research, New York, v.10, n.1, p.26-35, 1995.

ROSEN, L.W., HOUGH, D.O. Pathogenic weight-control behaviors of female college gymnasts. The Physician and Sportsmedicine, v.16, n.9, p.141-144, 1988.

ROWLAND, T.W., KELLEHER, J.F. Iron deficiency in athlete. American Journal of Diseases of Children, Chicago, v.143, n.2, p.197-200, 1989.

RUUD, J.S., GRANDJEAN, A.C. Preocupações Nutricionais das Atletas. In: WOLINSKY, I., HICKSON JR,J.F. Nutrição no exercício e no esporte. 2.ed. São Paulo : Roca, 1996. p.379-398.

SHORT, S.H. Estudos de consumo dietético e conhecimento de nutrição dos atletas e seus treinadores. In: WOLINSKY, I., HICKSON JR, J.F. Nutrição no exercício e no esporte. 2.ed. São Paulo: Roca, 1996. p. 401-443.

SKOLNICK, A.A. "Female athlete triad" Risk for women (news). Jama, Chicago, v.270, n.8, p.921-923, 1993.

SIMOPOULOS, A.P. Nutrition and fitness from the first Olympiad in $776 \mathrm{BC}$ to $393 \mathrm{AD}$ and the concept of positive health. American Journal of Clinical Nutrition, Bethesda, v.49, p.921-926, 1989. Supplement.

SNEAD, D.B., STUBBS, C.C., WELTMAN, J.Y., EVANS, W.S., VELDHUIS, J.D., ROGOL, A.D., TEATES, C.D., WELTMAN, A. Dietary patterns, eating behaviors, and bone mineral density in women runners. American Journal of Clinical Nutrition, Bethesda, v.56, n.4, p.705-711, 1992.

SOARES, E.A., BURINI, R.C., ISHII, M. Estudo antropométrico e dietético de nadadores competitivos: de áreas metropolitanas da Região Sudeste do Brasil. Revista de Saúde Pública, São Paulo, v.28, n.17, p.9-19, 1994.
SUNDGOT-BORGEN, J. Eating disorders in female athletes. Sports Medicine, Auckland, v.17, n.3, p.176-188, 1994a.

SUNDGOT-BORGEN, J. Risk and trigger factors for the development of eating disorders in female elite athletes. Medicine and Science in Sports and Exercise, Baltimore, v.26, n.4, p.414-419, $1994 b$.

SUNDGOT-BORGEN, J., CORBIN, C.B. Eating disorders among female athletes. The Physician and Sportsmedicine, v.15, n.2, p.89-95, 1987.

TELFORD, R.D., CUNNINGHAM, R.B., DEAKIN,V., KERR, D.A. Iron status and diet in athletes. Medicine and Science in Sports and Exercise, Baltimore, v.25, n.7, p.796-800, 1993.

TOFLER, I.R., STRYER, B.K., MICHELI, L.J., HERMAN, L,R. Physical and emotional problems of elite female gymnasts. New England Journal of Medicine, Boston, v.335, n.4, p.281-283, 1996.

VOSS, L.A., FADALE, P.D., HULSTYN, M.J. Exercise: induced loss of bone density in athletes. Journal of American Academy Orthopedic Surgery, v.6 , n.6, p.349-57. 1998.

VUORI, I. Peak bone mass and physical activity: a short review. Nutrition Reviews, New York, v.54, n.4, p.11-14, 1996.

WARREN, M.P. Clinical review 40: amenorrhea in endurance runners. Journal of Clinical Endocrinology and Metabolism, v.75, n.6, p.1393-1397, 1992.

WEIGHT, L.M., KLEIN,M, NOAKES, T.D., JACOBS, P. Sports Anemia: a real or apparent phenomenon in endurance-trained athletes? International Journal of Sports Medicine, Stuttgart, v.13, n.4, p.344-347,1992.

WICHMANN, S., MARTIN, D.R. Eating disorder in athletes. The Physician and Sportsmedicine, v.21, n.5, p.126-135, 1993.

WILLIAMS, S.R. Nutrition and diet therapy. 6.ed. St. Louis : Times, 1989. 969p. Chapter 18: Nutrition and physical fitness. p.538-561.

WILMORE, J.H. Eating and weight disorders in the female athlete. International Journal of Sport Nutrition, Champaign, v.1, n.2, p.104-117, 1991.

WILMORE, J.H., WAMBSGANS, K,C., BRENNER, M., BROEDER, C.E., PAIJMANS, I., VOLPE, J.A., WILMORE, K. M. Is there energy conservation in amenorrheic compared with eumenorrheic distance runners? Journal of Applied Physiology, Bethesda, v.72, n.1, p.15-22, 1992.

YEAGER, K.K., AGOSTINI, R., NATTIV, A.\& DRINKWATER, B. The female athlete triad: disordered eating, amenorrhea, osteoporosis. Medicine and Science in Sports and Exercise, Baltimore, v.25, n.7, p.775-777, 1993.

YURTH, E.F. Female athlete triad. The Western Journal of Medicine, San Francisco, v.162, n.2, p.149-150, 1995.

ZUCKER, P., AVENER, J., BAYDER, S., BROTMAN, A., MOORE, K., ZIMMERMAN, J. Eating disorders in young athletes. The Physician and Sportsmedicine, v.13, n.11, p.89-106, 1985.

Recebido para publicação em 23 de fevereiro de 1999 e aceito em 12 de setembro de 2000 . 